\title{
Interreader reproducibility of the Neck Imaging Reporting and Data system (NI- RADS) lexicon for the detection of residual/ recurrent disease in treated head and neck squamous cell carcinoma (HNSCC)
}

\author{
Tougan Taha Abdelaziz ${ }^{* *}$, Ahmed Abdel Khalek Abdel Razk ${ }^{2}$, Manar Maamoun Mohamed Ashour ${ }^{1}$ and
} Ahmed S. Abdelrahman ${ }^{1}$

\begin{abstract}
Background: To evaluate the inter- and intrareader agreement and reproducibility of the NI-RADS scoring system and lexicon with contrast-enhanced computed tomography (CECT) and contrast-enhanced magnetic resonance imaging (CEMRI).

Methods: This retrospective study included 97 CECT and CEMRI scans from 58 treated cases of head and neck squamous cell carcinoma (HNSCC) after the exclusion of head and neck cancers (HNCs) other than SCC and noncontrast and poor quality CT and MRI scans, with a total of 111 primary targets and 124 lymph node (LN) targets. Two experienced readers independently scored the likelihood of residual/recurrence for these targets based on the NI-RADS criteria and filled in report templates for NI-RADS lexicon diagnostic features. Inter- and intraobserver reproducibility was assessed with Cohen's kappa, and the percent agreement was calculated.

Results: Almost perfect interreader agreement was found for the final NI-RADS category of the primary lesions and $L N s$, with $K=0.808$ and 0.806 , respectively. Better agreement was found for $C T$ than for MRI $K=0.843$ and 0.77 , respectively, $P$ value 0.001). There was almost perfect agreement for excluding tissue enhancement $(K=0.826,95 \%$ $\mathrm{Cl}=0.658-0.993, P$ value 0.001$)$, with a percent agreement of $96.4 \%$, and substantial agreement for discrete nodular and diffuse mucosal enhancement $(K=0.826,95 \% \mathrm{Cl}=0.658-0.993, P$ value 0.001$)$, with a percent agreement of 96.4\%. There was fair agreement for focal mucosal nonmass and deep ill-defined enhancement. The intrareader agreement was almost perfect for most of the rated features ( $K$ ranging from 0.802 to 1 ), with the exception of enlarging discrete nodule/mass and focal mucosal nonmass-like enhancement, which had substantial intraobserver agreement (K ranging from 0.768 to 0.786 ).
\end{abstract}

Conclusion: The individual features of NI-RADS show variable degrees of confidence; however, the overall NI-RADS category was not significantly affected.

Keywords: NI-RADS lexicon, HNSCC, Cancer imaging, Inter-reader agreement

\footnotetext{
*Correspondence: toganwafi@yahoo.com

'Department of diagnostic radiology, faculty of medicine, Ain Shams University, Cairo, Egypt

Full list of author information is available at the end of the article
}

\section{$\triangle B M C$}

(C) The Author(s). 2020 Open Access This article is licensed under a Creative Commons Attribution 4.0 International License, which permits use, sharing, adaptation, distribution and reproduction in any medium or format, as long as you give appropriate credit to the original author(s) and the source, provide a link to the Creative Commons licence, and indicate if changes were made. The images or other third party material in this article are included in the article's Creative Commons licence, unless indicated otherwise in a credit line to the material. If material is not included in the article's Creative Commons licence and your intended use is not permitted by statutory regulation or exceeds the permitted use, you will need to obtain permission directly from the copyright holder. To view a copy of this licence, visit http://creativecommons.org/licenses/by/4.0/ The Creative Commons Public Domain Dedication waiver (http://creativecommons.org/publicdomain/zero/1.0/) applies to the data made available in this article, unless otherwise stated in a credit line to the data. 


\section{Background}

Locoregional head and neck squamous cell carcinoma (HNSCC) recurrence is observed in $15-50 \%$ of patients and represents a central cause of disease morbidity and mortality [1]. Disfigurement of the normal anatomy and the soft tissue changes occurring mainly after treatment with surgery and radiotherapy complicate the interpretation of imaging findings [2]. The free-text reporting method varies by radiologists' experience level and personal preference, a factor that may not answer modern clinical inquiries [3]. The imaging surveillance protocol for HNC varies among different institutes, and the radiologist's impression is frequently uncertain and dissociated from the management recommendation. In addition, the interobserver agreement between radiologists regarding tumor recurrence and appropriate follow-up is unknown. In view of these obstacles, the American College of Radiology (ACR) released NIRADS, a standardized report template associated with management recommendations [4]. This structured reporting approach can be simplified as a common language between radiologists and clinicians and a datadriven optimization of HNC imaging, with profitable results for patient management [5]. Furthermore, this reporting system serves in sharing data among different institutions, which may improve the research field in HNC [6].

There are limited but encouraging data concerning the baseline performance of NI-RADS for the evaluation of disease persistence/recurrence, interreader agreement, imaging modalities, and different time points of the surveillance protocol [7]. Although NI-RADS is currently adopted by several institutions, there is still limited evidence of whether the elaborated NI-RADS improves interreader agreement. In our opinion, structured reports should be a dynamic system, developed as the product of existing data and expert radiological and clinical consensus that will continue to be refined and updated as experience and validation data accumulate and in response to user feedback. To address this gap in knowledge, we designed this retrospective study involving CECT and CEMRI scans from treated patients with HNSCC.

The purpose of this study is to scrutinize the interand intrareader agreement of NI-RADS scoring for the likelihood of tumor residual/recurrence and to assess the interpretation reproducibility of NI-RADS lexicon imaging features using CECT and CEMRI in routine clinical practice.

\section{Methods}

\section{Patients}

Our institution ethics committee approved this singlecenter, retrospective study and waived the requirement for informed consent. Data were retrieved from medical records and PACS. The inclusion criteria were patients with HNSCC who finished their treatment and had been submitted to posttreatment imaging surveillance with either CECT or CEMRI according to our institution protocol, with no sex predilection. The exclusion criteria included HNCs other than SCC, scans other than CECT or CEMRI, scans not fulfilling the NI-RADS template requirements such as noncontrast-enhanced scans, and lowquality scans. An electronic search was performed in our hospital PACS system for the period from November 2017 to April 2019, yielding 500 CECT and 200 CEMRI examinations. A total of 97 scans from 58 patients met our inclusion and exclusion criteria, and these scans consisted of $45 \mathrm{CT}$ and $52 \mathrm{MRI}$ examinations. The following data were collected from the hospital medical records: demographic data, primary tumor site, initial stage (primary lesion, nodes and metastasis), and received treatment (chemotherapy, radiation, and surgery). Our institution posttreatment imaging surveillance protocol is a baseline scan 8 weeks after finishing treatment, followed by three-month follow-up intervals for 2 years.

\section{Image acquisition}

i. CECT: Multidetector spiral CT, 128-row MDCT scanner (GE 128, Optima 660, USA), supine position, with arms down. FOV: $28 \mathrm{~cm}$. One hundred milliliters of iodinated contrast agent was injected at a rate of $1-1.5 \mathrm{ml} / \mathrm{sec}$, and scanning was initiated $80-100 \mathrm{~s}$ after the start of the contrast agent injection. Axial images ranged from the frontal sinuses down through the mediastinum. The native CT images were acquired with a slice thickness of 0.6$0.75 \mathrm{~mm}$ and reformatted for display with a slice thickness of $3 \mathrm{~mm}$.

ii. CEMRI: 1.5 T MRI (Philips Ingenia scanner, Healthcare, Netherlands), using a dedicated head and neck coil. FOV AP 230, 4-mm slice thickness. Sequences: axial echo-planar DWI, precontrast axial T1WIs (TE/TR: 21/633 ms), coronal T1 (TE/TR: 14/555 ms), axial T2WIs (TE/TR: 10/7039 ms), sagittal T2WI (TE/TR: 100/3196.7 ms), and coronal T2 STIR $(T R / T I=3500 / 150, T E=80 \mathrm{~ms})$. Gadolinium $(0.1 \mathrm{mmol} / \mathrm{kg})$ was administered by using an injector with a flow rate of $2-3 \mathrm{ml} / \mathrm{sec}$, which was followed by postcontrast T1WI with fat saturation in the axial (TR/TE: 611/21 ms), sagittal (TR/TE: 570/14 ms), and coronal (TR/TE: 570/14 ms) planes; total scan time: $18-25 \mathrm{~min}$.

\section{Imaging analysis}

The initial scan interpretation was performed by a 25 year experienced reader and yielded a total of 235 
imaging targets: 111 primary targets and 124 LN targets. For precise interpretation, in cases of more than solitary, separate lesions, whether primary or nodal, lesions were defined with different numbers as primary target one and primary target two. Two head and neck radiologists with 15 and 11 years of experience served as reader 1 (R1) and reader 2 (R2). The readers had access to the patients' initial scans performed before treatment and the patients' demographic data, including age, sex, primary tumor site and the received treatment but were blinded to the patients' names and the original imaging interpretation. The readers were directed to the targets through a series/image number, primary tumor site, and additional spatial identifying information if multiple lesions were present. DWI images were excluded from interpretation to solely assess the defined NI-RADS lexicon diagnostic features.

Per lesion agreement and per patient agreement were assessed by asking the readers to fill in a template for the enhancement and morphological features of each target using the NI-RADS lexicon CEMRI and CECT diagnostic features and to categorize the final NI-RADS score for the primary site and cervical LNs separately.

We used the ACR NI-RADS reporting template directions as follows: category 1 for no evidence of recurrence (Fig. 1); category 2 for low suspicion in the form of an ill-defined nonmass-like area or nondifferential enhancement (Figs. 2 and 3); category 3 for highly suspicious lesions with discrete, enlarging or new lesions with differential enhancement (Fig. 4); and category 4 for definite clinical or radiological progression (Figs. 5 and 6) [7]. In nodal disease, NI-RADS 1 showed no enlarging LNs and no new suspicious features (Fig. 2). A growing LN was categorized as NI-RADS 2 if there was no morphological abnormality (Fig. 5) and NI-RADS 3 (Figs. 4 and 6) if a morphological abnormality was present (necrosis or extranodal extension) [5]. NI-RADS scoring for the primary tumor site and cervical LNs were documented. The exact reporting system was repeated after 4 months by the two readers. The same target lesions were arranged in a newly randomized way to avoid bias from the previous results.

\section{Statistical analysis}

The data were input into the computer and analyzed using Statistical Package for Social Science (IBM Corp, released 2013; IBM SPSS statistics for Windows, V. 22.0; Armonk, NY, USA) and GraphPad QuickCalcs (https:// www.grapghpad.com/quickcalcs/kappa1/). Qualitative data are described using numbers and percentages.

Binary variables were used for the morphological and enhancement categorical data, and an ordinal variable was used for the NI-RADS categorical data score. Crosstabulation for the categorical variables was performed with an estimation of the frequency of each categorical variable. The percent of agreement was estimated as a percent of concordance. Cohen's kappa coefficient $(\mathrm{k})$ was calculated to test the interreader and intrareader agreement and the $95 \%$ confidence intervals were calculated. The kappa test was statistically significant when the $p$ value was less than 0.05 . Kappa agreement was interpreted as follows: $0.01-0.20$ : slight agreement; $0.21-$ 0.40: fair agreement; 0.41-0.60: moderate agreement; 0.61-0.80: substantial agreement; and 0.81-0.99: almost perfect agreement).

\section{Results}

A total of 97 scans from fifty-eight treated HNSCC patients comprising $45 \mathrm{CT}$ scans and $52 \mathrm{MRI}$ scans were examined. These scans were performed at different time points within the initial 2 years of posttreatment followup. The tumor subsets of the included patients were 39 oral cavity carcinomas, 38 laryngeal carcinomas, 10 nasopharyngeal carcinomas, 5 sinonasal carcinomas, 3

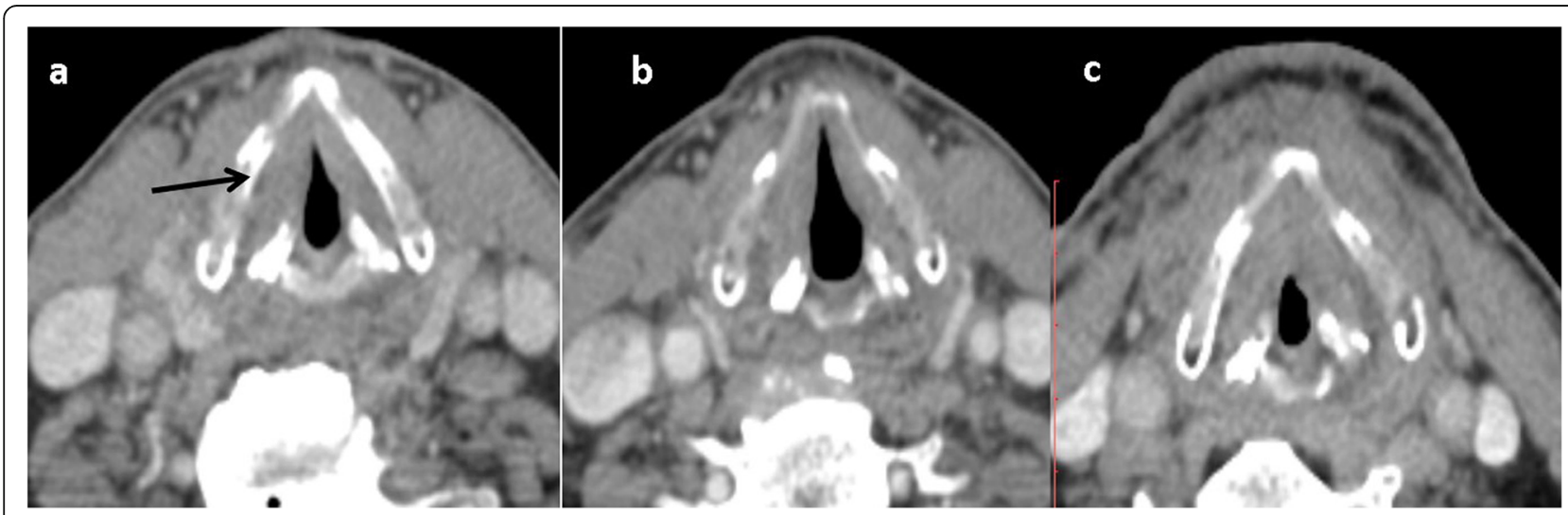

Fig. 1 Primary NI-RADS 1, Neck NI-RADS 1 of cancer larynx. a Pretreatment axial CECT shows right glottic SCC. b First post treatment CT follow up after 3 months shows mild, smooth thickening of the Right vocal cord. c Second follow up after another 3 months shows progressive diffuse glottic thickening with minimal mucosal enhancement. No cervical LN. The patient was stable on his further follow up 


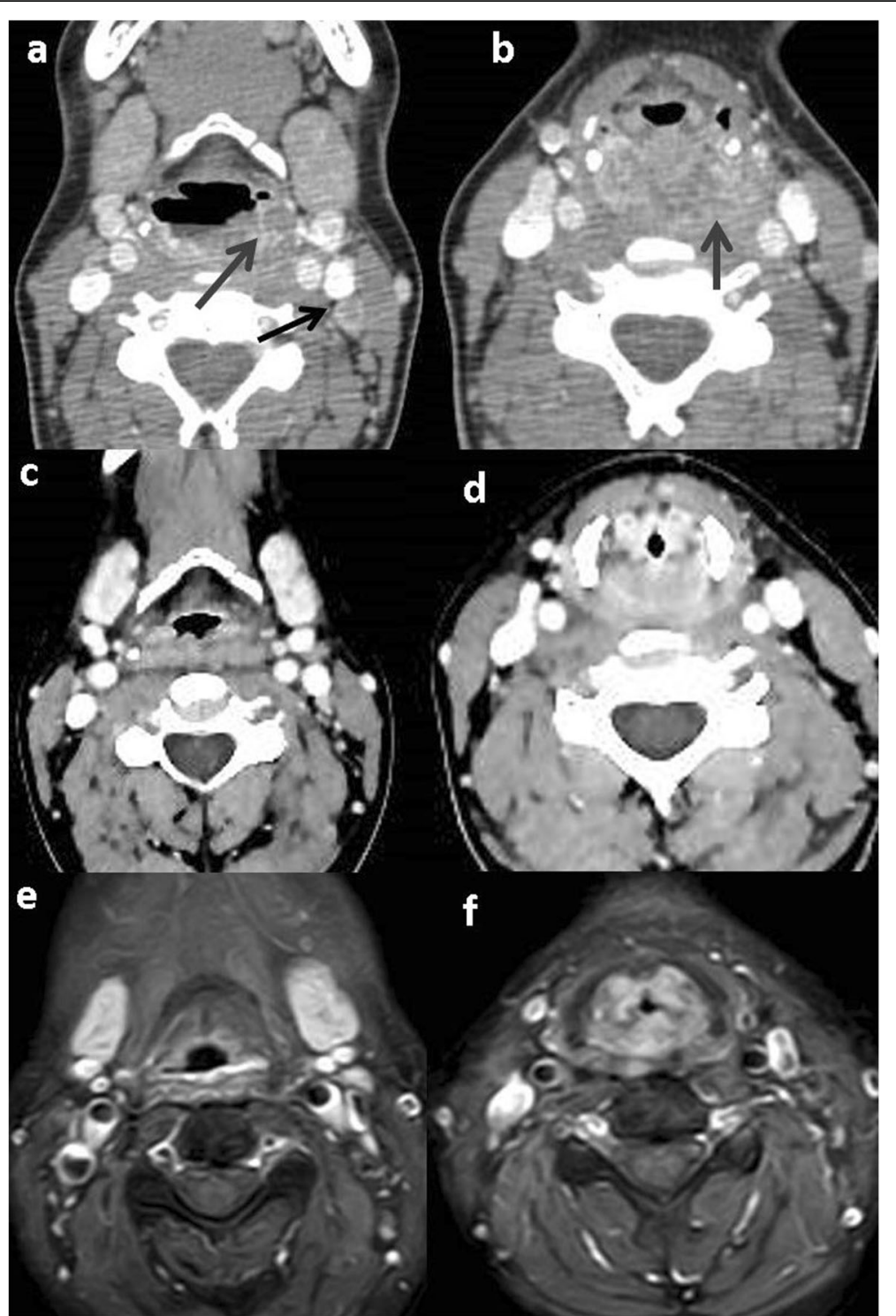

Fig. 2 Primary NI-RADS 2, Neck NI-RADS 1 hypopharyngeal carcinoma: a, b Pre-treatment axial CECT shows hypopharyngeal SCC (grey arrows) Left malignant LN with cystic changes (black arrow). c, d first post treatment follow up CT shows diffuse hypopharyngeal thickening and enhancement. Resolution of the $L N \mathbf{e}, \mathbf{f}$ Second follow up CEMRI axial images reveal stability of the Enhancement pattern

hypopharyngeal carcinomas, 1 skin neoplastic lesion, and 1 sublingual salivary gland carcinoma. Regarding patient demographics, $38(65.5 \%)$ patients were female and $20(34.5 \%)$ patients were male, with a mean age of $52.66 \pm 13.75$ years (range 18 to 89 years).

In this study, we analyzed the per lesion agreement for NI-RADS lexicon features for the included primary and nodal targets, and we found almost perfect agreement between the two observers in excluding any enhancement of the primary neck lesion, with $K=0.826$, (CI 95\%, 0.658-0.993) ( $P$ value $<0.001)$ and a percent agreement of $96.4 \%$, yet a substantial agreement was found in detection of either discrete nodular enhancement or diffuse linear mucosal enhancement, with $K=0.730$ and 0.706 , respectively $(P$ value $<0.001)$. Regarding the detection of either focal mucosal nonmass-like enhancement or deep illdefined enhancement, our study showed fair interobserver agreement $(K=0.309$ and 0.247 , respectively) (Table 1).

For the morphological features, we found moderate interobserver agreement for definite progressive mass lesions, low-density mucosal edema and nonmass-like tissue distortion, with $K=0.564,0.546$ and 0.402 , respectively $(P$ value $<0.001)$, yet much less agreement was found between the two readers in the detection of deep, ill-defined nondiscrete soft tissue, with $K=0.135$ $(P$ value $<0.049)$ (Table 1$)$. 


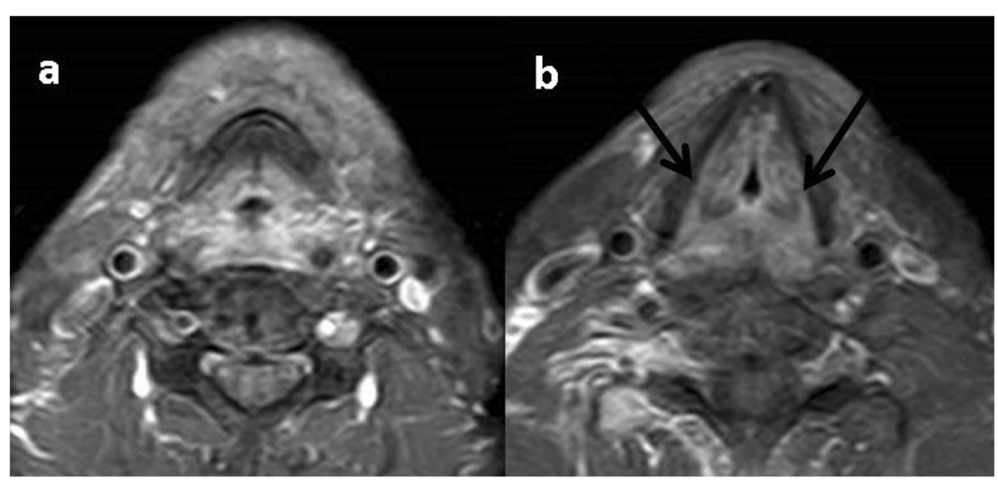

Fig. 3 Primary NI-RADS 2, Neck NI-RADS 1 treated glottic SCC: axial CE T1WI (a) supra glottic (b) glottic larynx show diffuse non-mass, mucosal and submucosal enhancement with no focal lesions. No cervical LN. The patient was stable on further follow ups

Almost perfect per lesion interobserver agreement was noted for the CT and MRI of the primary and neck LN lesions, with $K=0.819$ and 0.848 , respectively (CI 95\%, $0.731-0.908$ and $0.761-0.935$, respectively, $P$ value $<$ 0.001) (Tables 2 and 3).

The per scan interobserver agreement of cross-sectional imaging including both CT and MRI examinations for the primary and lymph node lesions was almost perfect, with $K=0.808$ and 0.806 , respectively (Tables 2 and 3 ).

Slightly less per lesion and per scan interobserver agreement was seen in the MRI examination of the primary neck lesions, with $K=0.778$ and 0.77 , respectively $(P$ value $<0.001)$, yet a higher agreement was seen in the CT examination, with $K=0.862$ and 0.843 , respectively $(P$ value $<0.001)$.
A near similar agreement was observed in the CT and MRI studies for the description of LNs regarding the per lesion and per scan analyses $(K=0.843$ and 0.867 for CT; $K=0.849$ and 0.816 for MRI; $P$ value $<0.001$ (Tables 2 and 3$)$.

Almost perfect interobserver agreement was found between the two readers for the discrimination of the oral cavity and laryngeal carcinoma, with $K=0.8$ and 0.92 , respectively (CI 95\%, $0.647-0.953$ and $0.817-1.0$, respectively, $P$ value $<0.001$ ), yet the agreement of other areas could not be calculated due to a reduced number of scans or missing primary NI-RADS categories (Table 2).

Substantial intraobserver agreement was found for both readers for the detection of new or enlarging

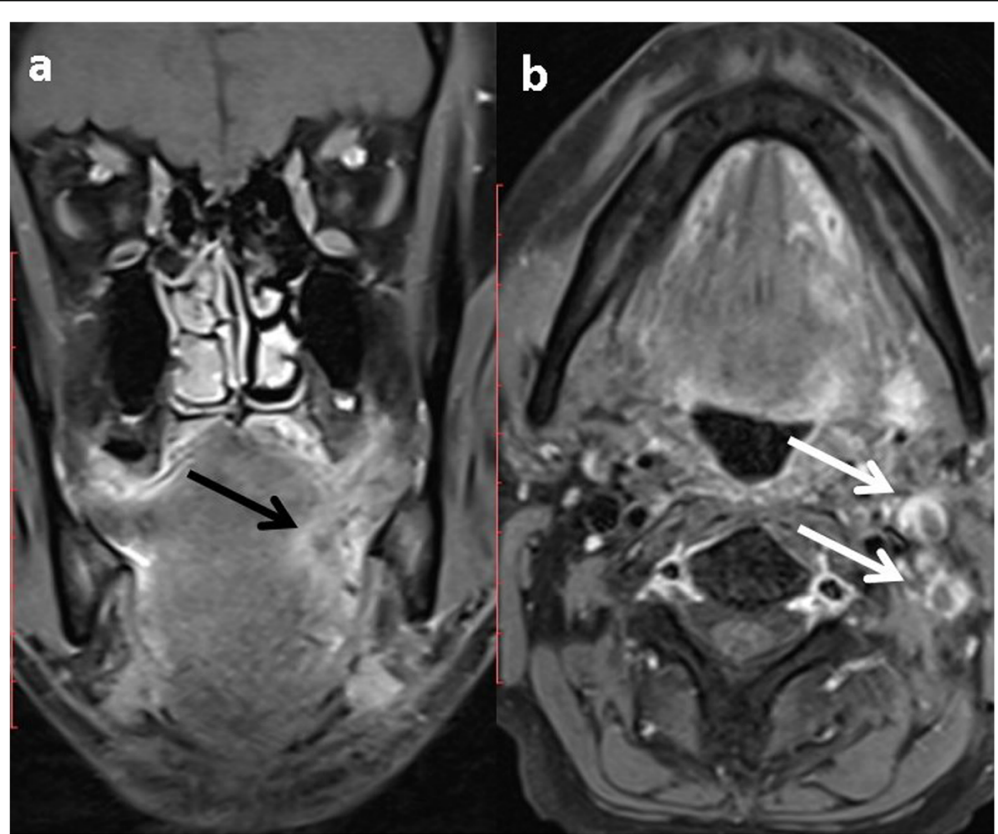

Fig. 4 Primary NI-RADS 3, Neck NI-RADS 3 of Tongue SCC: post treatment CEMRI, coronal (a) and axial (b) show highly suspicious focal enhancing mass at left lateral tongue (black arrow). Left level II malignant looking LN with central cystic breaking down (white arrows) 


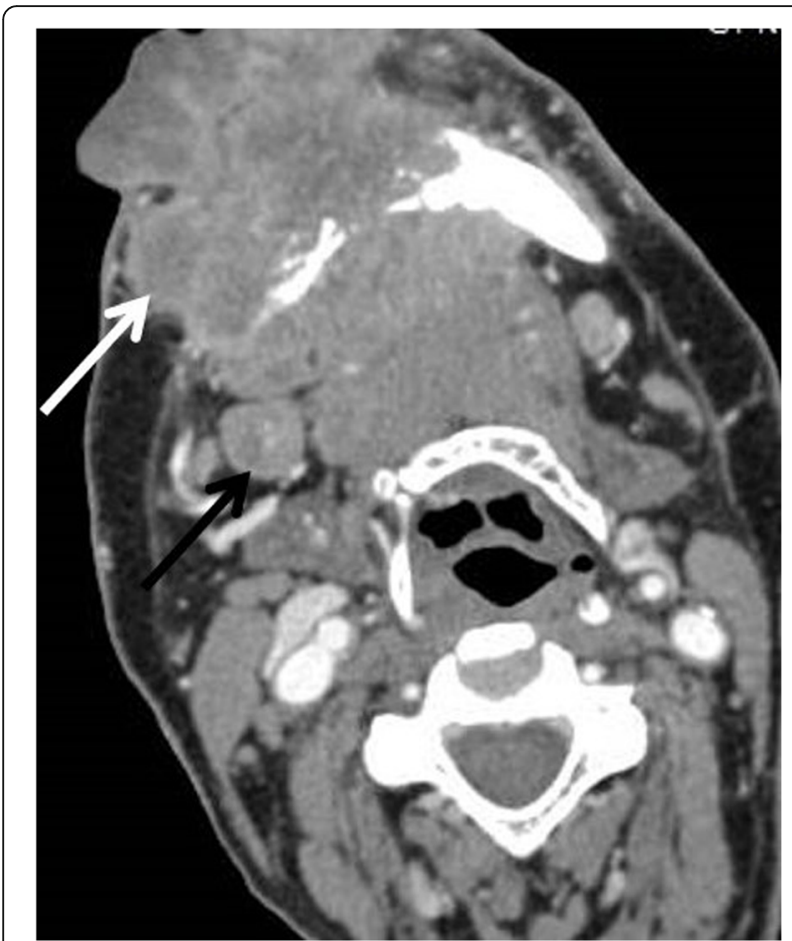

Fig. 5 Primary NI-RADS 4, Neck NI-RADS 2 of lip SCC, post treatment CECT scan shows aggressive soft tissue mass invading the mandible with skin invasion (white arrows). Right level II LN with no definite suspicious morphology (Black arrow)

discrete nodule/mass morphology, with $K=0.776$ and 0.768 (CI 95\%, 0.650-0.911 and 0.644-0.891, $P$ value $<0.001$ ) and a percent agreement of 90.1 and $89.2 \%$ for R1 and R2, respectively (Tables 4 and 5).

R2 (less experienced reader) showed a substantial intraobserver agreement for the discrimination of the primary neck lesion by CT examination and for the detection of the enhancement pattern of focal mucosal nonmass like enhancement, with $K=0.783$ and 0.786 and a percent agreement of 84.5 and $97.3 \%$, respectively ( $P$ value $<0.001$ ), yet $\mathrm{R} 1$ showed substantial intraobserver agreement for the distinction of $\mathrm{LN}$ lesions in the MRI examination, with $K=0.755(P$ value $<0.001)$ and a percent agreement of $83.9 \%$.

The two readers showed an almost perfect intraobserver agreement regarding the other rated features, with $\mathrm{K}$ values ranging from 0.802 to 1 and percent agreement values ranging from 86.6 to $100 \%$.

\section{Discussion}

The advances in treatment options and salvage procedures make free-text radiological reports no longer suitable for modern clinical inquiries. Structured reports have become a must to unify the radiological language and fulfill the key data elements, the quantified parameters, and the clinicians' inquires [8]. A structured reporting system allows the assimilation of relevant information and recommendations contingent upon the currently available literature, and the incorporation of these data with clinical and radiological findings not only allows a precise diagnosis but also affects further clinical management decisions [9].

The identification of recurrent superficial growing tumors could be problematic in CT and MRI; however, these tumors could be easily detected by clinical examination where imaging is required to evaluate the depth of tumor invasion. CT and MRI are more valuable in the detection of tumor recurrence in head and neck regions that are not completely convenient by clinical examination. An enhancing and infiltrating soft tissue mass is

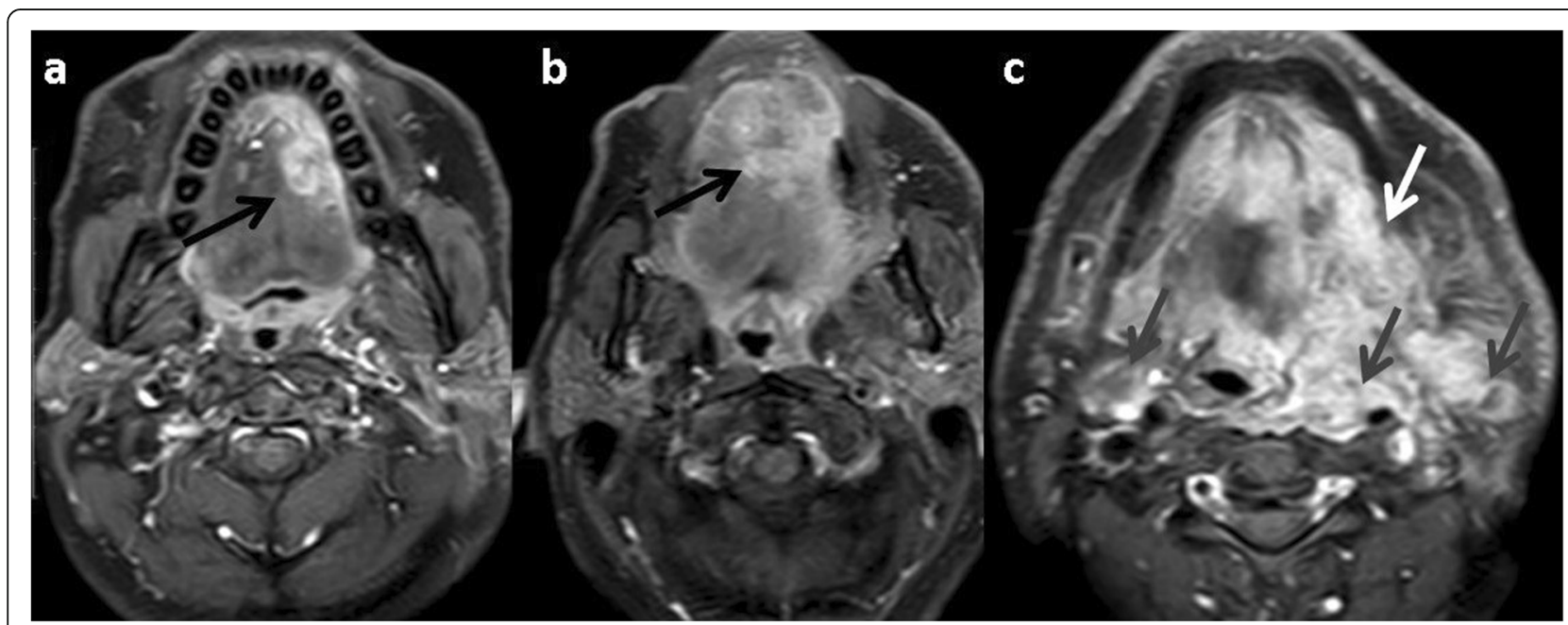

Fig. 6 Primary NI-RADS 4, Neck NI-RADS 3 for tongue SCC: axial CEMRI (a) pretreatment, (b) first FU (c) second FU. Show the continuous progression of the left lateral tongue enhancing mass, crossing the midline in the first follow up (black arrows), and invading the floor of the mouth and pharyngeal wall in second follow up (white arrows). De novo bilateral malignant looking LN (grey arrows) 
Table 1 Inter-observer agreement of the morphological findings for the 111 primary tumor sites

\begin{tabular}{|c|c|c|c|c|c|c|c|}
\hline Morphological and enhancement pattern & Reader 1 & Reader 2 & $\begin{array}{l}\text { Matched cases } \\
\text { between reader } 1 \& 2\end{array}$ & K & $95 \% \mathrm{Cl}$ & $\boldsymbol{P}$ value & Percent agreement \\
\hline Discrete nodular enhancement. & 53 & 56 & 47 & 0.730 & $0.603-0.857$ & $<0.001^{*}$ & $86.5 \%$ \\
\hline Definite progressive mass. & 25 & 13 & 12 & 0.564 & $0.369-0.760$ & $<0.001^{*}$ & $87.39 \%$ \\
\hline New or enlarging discrete nodule/ mass & 34 & 40 & 19 & 0.273 & $0.087-0.458$ & $0.004^{*}$ & $67.57 \%$ \\
\hline Deep ill-defined enhancement. & 63 & 24 & 21 & 0.247 & $0.116-0.378$ & $0.001^{*}$ & $59.46 \%$ \\
\hline Deep, ill-defined soft tissue mass. & 59 & 19 & 14 & 0.135 & $0.004-0.266$ & $0.049^{*}$ & $54.95 \%$ \\
\hline Focal mucosal non-mass like enhancement. & 16 & 6 & 4 & 0.309 & $0.05-0.568$ & $<0.001^{*}$ & $87.39 \%$ \\
\hline No enhancement. & 14 & 12 & 11 & 0.826 & $0.658-0.993$ & $<0.001^{*}$ & $96.4 \%$ \\
\hline Diffuse linear mucosal enhancement. & 31 & 30 & 24 & 0.706 & $0.56-0.856$ & $<0.001^{*}$ & $88.3 \%$ \\
\hline Non-mass like tissue distortion. & 67 & 49 & 41 & 0.402 & $0.242-0.562$ & $<0.001^{*}$ & $69.37 \%$ \\
\hline Low density mucoid mucosal edema. & 22 & 22 & 14 & 0.546 & $0.350-0.743$ & $<0.001^{*}$ & $85.6 \%$ \\
\hline
\end{tabular}

the established imaging finding for positive head and neck tumor residual/recurrence [10]; however, in clinical practice, not all patients present with these obvious diagnostic features, and different patterns of morphological tissue abnormalities and enhancement appear, which should be categorized as recurrent disease or posttreatment changes. The combination of morphology and enhancement patterns in addition to the rate of growth is integral to imaging algorithms. NI-RADS promotes standardization and therefore reproducibility across radiologists.

To our knowledge, few studies have evaluated the recently introduced NI-RADS [6, 7]; however, these few different studies have assessed the accuracy and overall performance of NI-RADS without introducing the NIRADS lexicon. Gaps in knowledge remain regarding the interreader agreement of individual features of the NIRADS lexicon and their integration into a structured algorithm. In this study, we categorized different tissue morphology and enhancement patterns according to the NI-RADS lexicon and evaluated the interreader agreement for the different features that affect the agreement for the lesion and therefore the overall NI-RADS category of the patient.
We assessed the NI-RADS interreader reliability for the CECT and CEMRI templates. NI-RADS was initially developed for surveillance with CECT and then adapted to PET/CT and was finally modified for CEMRI scans. In our institution, the choice of imaging modality depends on the tumor site to be evaluated, the proposed clinical inquiries, and the patients' clinical status in an individualized manner.

Considering the enhancement patterns, our study revealed significant interreader agreement for excluding tissue enhancement, the detection of discrete nodules, and linear mucosal enhancement patterns. However, the readers were skeptical about focal mucosal nonmass and deep ill-defined enhancement patterns. In a previous study, Nooij et al. reported that the most controversy for the primary site occurs when both the recurrent tumor and the treatment-induced inflammation show a high T2 signal and postcontrast enhancement [11].

In the current study, the morphological features of NIRADS showed a lower degree of confidence for nonmass like tissue distortion and deep ill-defined soft tissue masses; this is likely due to the overlap between recurrent tumor and posttreatment changes or benign complications. Recent studies have described the potential

Table 2 Inter-observer agreement of the CT \& MRI \& cross section examination per lesion (P lesion) and per patient (P patient) for the primary tumor site

\begin{tabular}{|c|c|c|c|c|}
\hline Groups & $K$ & $95 \% \mathrm{Cl}$ & $\boldsymbol{P}$ value & Percent agreement \\
\hline P lesion: $\mathrm{CT}(\mathrm{No}=51)$ & 0.862 & $0.748-0.977$ & $<0.001^{*}$ & $90.2 \%$ \\
\hline P lesion: $M R I(\mathrm{No}=60)$ & 0.778 & $0.645-0.912$ & $<0.001^{*}$ & $85.0 \%$ \\
\hline P lesion: Cross section $(\mathrm{No}=111)$ & 0.819 & $0.731-0.908$ & $<0.001^{*}$ & $87.39 \%$ \\
\hline P lesion for oral cavity: Cross section $(\mathrm{No}=39)$ & 0.80 & $0.647-0.953$ & $<0.001^{*}$ & $82.1 \%$ \\
\hline P lesion for larynx: Cross section $(\mathrm{No}=38)$ & 0.92 & $0.817-1.0$ & $<0.001^{*}$ & $94.7 \%$ \\
\hline P patient: $C T(\mathrm{No}=45)$ & 0.843 & $0.713-0.973$ & $<0.001^{*}$ & $88.9 \%$ \\
\hline$P$ patient: $\mathrm{MRI}(\mathrm{No}=52)$ & 0.77 & $0.627-0.917$ & $<0.001^{*}$ & $84.62 \%$ \\
\hline P patient: Cross section $(\mathrm{No}=97)$ & 0.808 & $0.710-0.904$ & $<0.001^{*}$ & $86.6 \%$ \\
\hline
\end{tabular}


Table 3 Inter-observer agreement of the CT \& MRI \& cross section examination per lesion (P lesion) and per patient scan (P patient) for $L N$

\begin{tabular}{lllll}
\hline Groups & $\boldsymbol{K}$ & $95 \% \mathrm{Cl}$ & $\boldsymbol{P}$ value & Percent agreement \\
\hline LN P lesion: CT (No= 55) & 0.843 & $0.695-0.991$ & $<0.001$ & $92.73 \%$ \\
LN P lesion: MRI (No= 69) & 0.849 & $0.734-0.965$ & $<0.001$ & $91.3 \%$ \\
LN P lesion: Cross section $(\mathbf{N o}=\mathbf{1 2 4 )}$ & 0.848 & $0.761-0.935$ & $<0.001$ & $91.94 \%$ \\
LN P patient: CT (No= 45) & 0.867 & $0.687-0.99$ & $<0.001$ & $95.6 \%$ \\
LN P patient: MRI $(\mathbf{N o}=\mathbf{5 2 )}$ & 0.816 & $0.642-0.989$ & $<0.001$ & $92.3 \%$ \\
LN P patient: Cross section $(\mathbf{N o}=\mathbf{9 7})$ & 0.806 & $0.668-0.945$ & $<0.001$ & $92.8 \%$ \\
\hline
\end{tabular}

role of functional imaging to see the overall picture of posttreatment changes. $M$. Lell et al. reported that using conventional features such as morphology and enhancement makes it difficult to differentiate a residual or recurrent tumor from posttreatment changes, especially in the early posttreatment interval, resulting in a $46 \%$ false positive diagnosis. They found that with longer followup intervals, the posttreatment changes decreased, and the diagnosis was more definite [10]. Another study by Van der Hoorn et al. described the limited role of conventional MRI in posttreatment evaluation, which had a sensitivity of $84 \%$ and a specificity of $82 \%$ for local treatment response evaluation [12]. For these reasons, NIRADS combines semiquantitative readouts based on observation recognition and quantitative measures. This combination led to a narrow change regarding the final NI-RADS category despite the varying levels of consensus for the individual features. In this study, there was almost perfect interreader agreement for the NI-RADS category for the primary tumor site, which was in agreement with the findings of a study performed by Krieger et al., which showed very good interobserver agreement of 0.821 (95\% CI, 0.657-0.986) with $P<0.001$ [7]. The other quantitative imaging biomarkers, such as diffusion, perfusion and spectroscopy, were not included in the NI-RADS algorithms.

The malignant criteria for nodal disease discussed in previous studies included increased axial diameter more than $10 \mathrm{~mm}$, morphological changes such as attaining a rounded shape, regional grouping, presence of necrosis,

Table 4 Intra-observer agreement for the morphological findings for the 111 primary lesions

\begin{tabular}{|c|c|c|c|c|c|c|c|c|}
\hline Morphological and enhancement pattern & Reader & First reading & $\begin{array}{l}\text { Second } \\
\text { reading }\end{array}$ & $\begin{array}{l}\text { Matched cases between } \\
\text { 1st \& 2nd reading }\end{array}$ & K & $95 \% \mathrm{Cl}$ & $\boldsymbol{P}$ value & $\begin{array}{l}\text { Percent } \\
\text { agreement }\end{array}$ \\
\hline \multirow[t]{2}{*}{ Discrete nodular enhancement. } & 1 & 53 & 53 & 48 & 0.819 & $0.713-0.925$ & $<0.001^{*}$ & $95.4 \%$ \\
\hline & 2 & 56 & 62 & 55 & 0.856 & $0.760-0.952$ & $<0.001^{*}$ & $92.7 \%$ \\
\hline \multirow[t]{2}{*}{ Definite progressive mass. } & 1 & 25 & 26 & 22 & 0.822 & $0.694-0.949$ & $<0.001^{*}$ & $93.7 \%$ \\
\hline & 2 & 13 & 15 & 12 & 0.837 & $0.682-0.992$ & $<0.001^{*}$ & $92.8 \%$ \\
\hline \multirow[t]{2}{*}{ New or enlarging discrete nodule mass } & 1 & 34 & 39 & 31 & 0.776 & $0.650-0.911$ & $<0.001^{*}$ & $90.1 \%$ \\
\hline & 2 & 40 & 42 & 35 & 0.768 & $0.644-0.891$ & $<0.001^{*}$ & $89.2 \%$ \\
\hline \multirow[t]{2}{*}{ Deep ill-defined enhancement. } & 1 & 63 & 56 & 54 & 0.802 & $0.795-0.983$ & $<0.001^{*}$ & $90 \%$ \\
\hline & 2 & 24 & 27 & 22 & 0.822 & $0.695-0.949$ & $<0.001^{*}$ & $93.7 \%$ \\
\hline \multirow[t]{2}{*}{ Deep, ill-defined soft tissue mass. } & 1 & 59 & 59 & 54 & 0.819 & $0.711-0.927$ & $<0.001^{*}$ & $90.9 \%$ \\
\hline & 2 & 19 & 23 & 18 & 0.824 & $0.688-0.959$ & $<0.001^{*}$ & $94.6 \%$ \\
\hline \multirow[t]{2}{*}{ Focal mucosal non-mass like enhancement. } & 1 & 16 & 19 & 15 & 0.831 & $0.687-0.974$ & $<0.001^{*}$ & $87.39 \%$ \\
\hline & 2 & 6 & 9 & 6 & 0.786 & $0.552-1.0$ & $<0.001^{*}$ & $97.3 \%$ \\
\hline \multirow[t]{2}{*}{ No enhancement. } & 1 & 14 & 14 & 14 & 1 & $1-1$ & $<0.001^{*}$ & $100 \%$ \\
\hline & 2 & 12 & 11 & 11 & 0.952 & $0.857-1.0$ & $<0.001^{*}$ & $99.1 \%$ \\
\hline \multirow[t]{2}{*}{ Diffuse linear mucosal enhancement. } & 1 & 31 & 32 & 29 & 0.889 & $0.794-0.983$ & $<0.001^{*}$ & $95.5 \%$ \\
\hline & 2 & 30 & 31 & 28 & 0.887 & $0.790-0.983$ & $<0.001^{*}$ & $95.5 \%$ \\
\hline \multirow[t]{2}{*}{ Non-mass like tissue distortion. } & 1 & 67 & 66 & 64 & 0.906 & $0.825-0.986$ & $<0.001^{*}$ & $95.5 \%$ \\
\hline & 2 & 49 & 49 & 45 & 0.854 & $0.756-0.952$ & $<0.001^{*}$ & $92.8 \%$ \\
\hline \multirow[t]{2}{*}{ Low density mucoid mucosal edema. } & 1 & 22 & 20 & 19 & 0.883 & $0.994-0.771$ & $<0.001^{*}$ & $96.4 \%$ \\
\hline & 2 & 22 & 22 & 19 & 0.830 & $0.698-0.961$ & $<0.001^{*}$ & $94.6 \%$ \\
\hline
\end{tabular}


Table 5 Intra-observer agreement of the CT \& MRI \& cross section examination per patient (P patient) for the primary tumor site and LN

\begin{tabular}{|c|c|c|c|c|c|}
\hline Groups & Reader & $K$ & $95 \% \mathrm{Cl}$ & $\boldsymbol{P}$ value & Percent agreement \\
\hline \multirow[t]{2}{*}{ P patient: $\mathrm{CT}(\mathrm{No}=45)$} & 1 & 0.813 & $0.676-0.950$ & $<0.001^{*}$ & $86.6 \%$ \\
\hline & 2 & 0.783 & $0.634-0.931$ & $<0.001^{*}$ & $84.5 \%$ \\
\hline \multirow[t]{2}{*}{$P$ patient: $\mathrm{MRI}(\mathrm{No}=52)$} & 1 & 0.915 & $0.820-1.0$ & $<0.001^{*}$ & $94.2 \%$ \\
\hline & 2 & 0.884 & $0.774-0.993$ & $<0.001^{*}$ & $92.2 \%$ \\
\hline \multirow[t]{2}{*}{ P patient: Cross section $(\mathrm{No}=97)$} & 1 & 0.867 & $0.784-0.949$ & $<0.001^{*}$ & $90.7 \%$ \\
\hline & 2 & 0.838 & $0.747-0.928$ & $<0.001^{*}$ & $88.6 \%$ \\
\hline \multirow[t]{2}{*}{ P lesion for oral cavity: Cross section $(\mathrm{No}=39)$} & 1 & 0.879 & $0.747-1.0$ & $<0.001^{*}$ & $92.3 \%$ \\
\hline & 2 & 0.836 & $0.685-0.986$ & $<0.001^{*}$ & $89.8 \%$ \\
\hline \multirow[t]{2}{*}{ P lesion for larynx: Cross section $(\mathrm{No}=38)$} & 1 & 0.883 & $0.757-1.0$ & $<0.001^{*}$ & $92.2 \%$ \\
\hline & 2 & 0.848 & $0.706-0.989$ & $<0.001^{*}$ & $89.5 \%$ \\
\hline \multirow[t]{2}{*}{ LN P patient Scan: CT $(\mathrm{No}=45)$} & 1 & 1 & $1-1$ & $<0.001^{*}$ & $100 \%$ \\
\hline & 2 & 0.873 & $0.710-1.0$ & $<0.001^{*}$ & $95.6 \%$ \\
\hline \multirow[t]{2}{*}{ LN P patient Scan: MRI $(\mathrm{No}=52)$} & 1 & 0.755 & $0.567-0.943$ & $<0.001^{*}$ & $83.9 \%$ \\
\hline & 2 & 0.834 & $0.681-0.986$ & $<0.001^{*}$ & $92.3 \%$ \\
\hline \multirow[t]{2}{*}{ LN P patient Scan: Cross section $(\mathrm{No}=97)$} & 1 & 0.860 & $0.744-0.975$ & $<0.001^{*}$ & $94.9 \%$ \\
\hline & 2 & 0.851 & $0.739-0.962$ & $<0.001^{*}$ & $93.9 \%$ \\
\hline
\end{tabular}

grouping, and extracapsular spread of the tumor [13]. Lymph node assessment is impeded by reactionary changes, as reactive LNs may show borderline features; moreover, normally sized nodes can still contain malignant infiltration. Using size criteria for the evaluation of $\mathrm{LN}$ infiltration is confusing and unreliable because there are multiple size criteria reported in the literature [14]. In a study performed by Aiken et al., they found that cross-sectional imaging has high specificity for extracapsular spread (88\%) and low sensitivity (68\%), and the presence of necrosis was the best predictor for extracapsular tumor spread $(p=0.001)$, which had a significant $P$ value compared with irregular border $(p=0.055)$ and gross invasion $(p=0.068)$ [15]. NI-RADS combines the size, morphology and functional features of cervical LNs in the follow-up algorithm. The current study revealed perfect per scan interobserver agreement for crosssectional imaging, including both CT and MRI examination for both primary and lymph node lesions.

MRI reverses biochemical tissue characteristics and could improve tissue resolution compared to CT. Detrimentally, the scan times are relatively long during which the patient must remain still. Modern multislice CT machines allow short scan times without motion artifacts, a precious advantage in HNC patients who may have difficulty breathing, swallowing secretions, and lying flat [16]. In this study, better interobserver agreement was found in CT for the per lesion and per scan evaluations of the primary site; however, it did not significantly exceed the MRI agreement. Furthermore, a near similar agreement between the two modalities was found for $\mathrm{LN}$ evaluation.
There is a large geographical difference regarding the incidence of the primary site of head and neck cancers, which varies according to the prevalence of risk factors, ethnic and genetic differences among populations, and environmental factors such as diet and lifestyle [17]. In the current study, the oral cavity and larynx were the most common primary sites with an almost perfect interobserver agreement for the different NI-RADS categories.

Finally, there was satisfactory agreement between the head and neck radiologists for the final NI-RADS categories, and the variability in the morphological features could be overcome by adding functional imaging such as $\mathrm{PET} / \mathrm{CT}$, which is already included in the NI-RADS algorithms. The other quantitative imaging biomarkers not included in NI-RADS show the potential to provide decision support tools in the management pathway of head and neck cancer.

\section{Limitations}

Our work encompassed all types of HNSCC collectively instead of investigating each subsite separately. Another limitation is the combination of subsequent CT and MRI scans in our study when ideally each modality should have been scrutinized separately. This study lacks functional imaging and an evaluation of the biological features of the treated tissue, which is expected to decrease the debate and increase the radiologist confidence about treated HNC imaging. The last limitation is the limited number of patients, as investigating a larger cohort of patients in a multicenter study is recommended. 


\section{Conclusion}

The interreader agreement was almost perfect for the final NI-RADS categorization of primary lesions and nodal disease. Different degrees of confidence were encountered regarding the morphology and enhancement features, which can be attributed to the overlap between posttreatment changes or benign complications and recurrent disease; however, this did not affect the overall NI-RADS category and the final recommendation for the next step in follow-up or clinical management.

\section{Abbreviations}

NI-RADS: Neck imaging reporting and data system; HNSCC: Head and neck squamous cell carcinoma; HNC: Head and neck cancer; ACR: American college of radiology; LN: Lymph node; CEMRI: Contrast enhanced magnetic resonance imaging; CECT: Contrast enhanced computed tomography

\section{Acknowledgements}

Not applicable.

\section{Authors' contributions}

TT: Idea of the research, Design of work, Reader 1, Manuscript writing. AS: Reader 2, Results \& statistics. MM: Collecting cases/ AA: Idea of the research, Design of work, Revising the manuscript. The author(s) read and approved the final manuscript.

\section{Funding}

This research was funded by any authority.

\section{Availability of data and materials}

Not applicable.

\section{Ethics approval and consent to participate}

This retrospective study was approved by our institution ethics committee, that waived the requirement for informed consent.

\section{Consent for publication}

Not applicable.

\section{Competing interests}

All the authors declare that they have no competing interest.

\section{Author details}

${ }^{1}$ Department of diagnostic radiology, faculty of medicine, Ain Shams University, Cairo, Egypt. ${ }^{2}$ Department of diagnostic radiology, faculty of medicine, Mansoura University, Mansoura, Egypt.

Received: 17 March 2020 Accepted: 4 August 2020

Published online: 18 August 2020

\section{References}

1. Chang J, Wu C, Yuan K, Wu A, Wu S. Locoregionally recurrent head and neck squamous cell carcinoma: incidence, survival, prognostic factors, and treatment outcomes. Oncotarget. 2017;8(33):55600-55612. https://doi.org/ 10.18632/oncotarget.16340

2. Gage K, Thomas K, Jeong D, Stallworth D, Arrington J. Multimodal imaging of head and neck squamous cell carcinoma. Cancer Control 2017;24(2):172197. https://doi.org/10.1177/107327481702400209.

3. Lambin P, Leijenaar R, Deist T, Peerlings J, de Jing E, Van Timmeren J. Radiomics: the bridge between medical imaging and personalized medicine. Nat Rev Clin Oncol 2017;14(12):749-762. https://doi.org/10.1038/ nrclinonc.2017.141

4. Aiken A, Farley A, Baugnon K, Dusak R, Beitler J, Hudgins P. Implementation of a novel surveillance template for head and neck Cancer: neck imaging reporting and data system (NI-RADS). J Am Coll Radiol 2016;13(6):743-746. https://doi.org/10.1016/j.jacr.2015.09.032.

5. Aiken A, Rath T, Anzai Y, Rath T, Hoang J, Wiggins R. ACR neck imaging reporting and data systems (NI-RADS): a white paper of the ACR NI-RADS committee. J Am Coll Radiol 2015;15(8):1097-1108. https://doi.org/10.1016/j. jacr.2018.05.006.

6. Wangaryattawanich $P$, Branstetter B, Hughes M, Clump D, Heron D, Rath T. Negative predictive value of NI-RADS category 2 in the first Posttreatment FDG-PET/CT in head and neck squamous cell carcinoma. AJNR Am J Neuroradiol. 2018;39(10):1884-8.

7. Krieger D, Hudgins P, Nayak G, Baugnon K, Corey A, Patel M, et al., Initial performance of NI-RADS to predict residual or recurrent head and neck squamous cell carcinoma. AJNR Am J Neuroradiol 2017;38(6):1193-1199. https://doi.org/10.3174/ajnr.A5157.

8. European Society of Radiology. ESR paper on structured reporting in radiology. Insights Imaging 2018:9:1-7. https://doi.org/10.1007/s13244-0170588-8.

9. Fowler K, Tang A, Santillan C, Bhargavan-Chatfield M, Heiken J, Jha R, et al. Inter-reader reliability of LI-RADS version 2014 algorithm and imaging features for diagnosis of hepatocellular carcinoma: a large international multireader study. Radiology. 2018;286(1):173-185. https://doi.org/10.1148/ radiol.2017170376.

10. Lell M, Baum U, Greess H, Nömayr A, Nkenke E, Koester M, et al. Head and neck tumors: imaging recurrent tumor and post-therapeutic changes with CT and MRI. Eur J Radiol 2000;33(3):239-247. https://doi.org/10.1016/s0720048x(99)00120-5.

11. Nooij R, Hof J, Jan Van Laar P, Van der Hoorn A. Functional MRI for treatment evaluation in patients with head and neck squamous cell carcinoma: a review of the literature from a radiologist perspective. Curr Radiol Rep. 2018;6(2). https://doi.org/10.1007/s40134-018-0262-z.

12. Van der Hoorn A, Van Laar P, Holtman G, Van der Hoorn A. Diagnostic accuracy of magnetic resonance imaging techniques for treatment response evaluation in patients with head and neck tumors, a systematic review and meta-analysis. PLoS One 2017;12(5):e0177986. https://doi.org/10. 1371/journal.pone.0177986.

13. Wu L, Xu J, Hua J, Gu H, Zhu J, Hu J. Value of diffusion-weighted MR imaging performed with quantitative apparent diffusion coefficient values for cervical lymphadenopathy. J Magn Reson Imaging 2013;38(3):663-670. https://doi.org/10.1002/jmri.24014.

14. Hoang J, Vanka J, Ludwig B, Glastonbury C. Evaluation of cervical lymph nodes in head and neck cancer with CT and MRI: tips, traps, and a systematic approach. Am J Roentgenol 2013;200:17-25. https://doi.org/10. 2214/AJR.12.8960

15. Aiken A, Poliashenko S, Beitler J. Accuracy of preoperative imaging in detecting nodal extracapsular spread in oral cavity squamous cell carcinoma. AJNR Am J Neuroradiol 2015;36(9):1776-1781. https://doi.org/10. 3174/ajnr.A4372

16. Lewis-Jones H, Colley S, Gibson G. Imaging in head and neck cancer: United Kingdom National Multidisciplinary Guidelines. J Laryngol Otol 2016;130: S28-S31. https://doi.org/10.1017/S0022215116000396.

17. Parasher A, Abramowitz M, Weed D, Franzmann E, Goodwin J, Hu J. Ethnicity and clinical outcomes in head and neck Cancer: an analysis of the SEER database. J Racial Ethn Health Disparities 2014;1:267-274. https://doi.org/10.1007/s40615-014-0033-3.

\section{Publisher's Note}

Springer Nature remains neutral with regard to jurisdictional claims in published maps and institutional affiliations.

\section{Ready to submit your research? Choose BMC and benefit from:}

- fast, convenient online submission

- thorough peer review by experienced researchers in your field

- rapid publication on acceptance

- support for research data, including large and complex data types

- gold Open Access which fosters wider collaboration and increased citations

- maximum visibility for your research: over $100 \mathrm{M}$ website views per year

At $\mathrm{BMC}$, research is always in progress.

Learn more biomedcentral.com/submissions 